\title{
SPACE-TIME CONVOLUTIONAL CODES OVER GF(p) FOR THE QUASI-STATIC, FLAT RAYLEIGH FADING CHANNEL
}

\author{
Mário de Noronha-Neto, Richard Demo Souza, and Bartolomeu F. Uchôa-Filho
}

\begin{abstract}
In this paper, we consider a space-time coded system consisting of a rate $R=1 / n$ linear convolutional encoder over $\mathrm{GF}(p), p$ a prime, followed by $n$ mappers from $\mathrm{GF}(p)$ into a $p$-ary signal constellation, and by a transmitter ith $n$ transmit antennas. At each time, the $n p$-ary coded $\$$ mbols are transmitted simultaneously from the $n$ antennas. The convolutional codes are designed to provide the best error performance in the quasi-static, flat Rayleigh fading channel, according to the rank and the determinant criteria derived by Tarokh et al. A spectral efficiency of $\log _{2}(p) \mathrm{b} / \mathrm{s} / \mathrm{Hz}$ $\therefore$ achieved. Simple conditions on the generator matrices of a rate $R=1 / 2$ convolutional code are given so that maximum diversity advantage is guaranteed. The linear structure of these convolutional codes reduces significantly the computer search effort with respect to the determinant criterion. In this regard, some properties of reciprocal STC's are derived. New space-time codes with two transmit antennas are presented for the 5-PSK and 7-PSK modulations, with spectral efficiencies 2.32 and $2.81 \mathrm{~b} / \mathrm{s} / \mathrm{Hz}$, respectively. Simulation results in the form of frame error rate versus signal-tonoise ratio are reported for these codes, showing agreement with the theoretical results.
\end{abstract}

Keywords: Diversity, fading channels, multiple transmit antennas, space-time convolutional codes, wireless communications.

Resumo - Neste trabalho consideramos um sistema codificado espácio-temporal composto por um codificador convolucional linear de taxa $R=1 / n$ sobre $\mathrm{GF}(p)$, onde $p$ é primo, seguido de $n$ mapeadores de símbolos em $G F(p)$ para uma constelação de sinais $p$-ários e de um transmissor contendo $n$ antenas transmissoras. Em cada instante de tempo, os $n$ símbolos $p$-ários codificados são transmitidos simultaneamente através das $n$ antenas. Os códigos convolucionais são projetados para proporcionar o melhor desempentל de erro em um canal com desvanecimento Rayleigh plano quase-estático, de acordo com os critérios do posto e do determinante derivados por Tarokh et al. Uma eficiência espectral de $\log _{2}(p) \mathrm{b} / \mathrm{s} / \mathrm{Hz}$ é alcançada. Restrições simples impostas às matrizes geradoras de um codificador convolucional de taxa $R=1 / 2$ são apresentadas para garantir ganho de diversidade máximo. A estrutura linear destes códigos re-

This work was supported by the Coordenação de Aperfeiçoamento de Pessoal de Nivel Superior (CAPES), as an M.Sc. scholarship, and by Conselho Nacional de Desenvolvimento Científico e Tecnológico (CNPq) under grants 141577/2001-0, 301593/96-5, and $+62450 / 00-7$.

The authors are with the Conmunications Research Group, Department of Electrical Engineering, Federal University of Santa Catarina - Florianópolis - SC, Brazil. E-mails: \{mnoronha,richard,uchoa\}@eel.ufsc.br ) duz significantemente o esforço da busca computacional com relação ao critério do determinante. Nesta direção, algumas propriedades de STC recíprocos são demonstradas. Novos códigos espácio-temporais com duas antenas transmissoras são apresentados para as modulações 5-PSK e 7-PSK com eficiências espectrais de 2.32 e $2.81 \mathrm{~b} / \mathrm{s} / \mathrm{Hz}$, respectivamente. Resultados de simulações na forma de taxa de erro de frame versus relação sinal-ruído são apresentados e estão de acordo com os resultados teóricos.

Palavras-chave: Diversidade, canais com desvanecimento, múltiplas antenas transmissoras, códigos convolucionais espácio-temporais, comunicações sem fio.

\section{INTRODUCTION}

In a recent wave of information theoretic results, Telatar [1], Foschini and Gans [2], and Marzetta and Hochwald [3] have demonstrated that the capacity of wireless communications systems employing multiple antennas is noticeably greater than the capacity of single-antenna systems for fading channels. Motivated by [1] and [2], Tarokh et al. [4] introduced the so-called "space-time coding," whereby redundancy is placed in an appropriate way across space (transmit antennas) as well as time to achieve coding and transmit diversity advantages simultaneously. Space-time coding has rapidly become the most accepted technique for providing reliable communications over fading channels employing multiple transmit antennas.

A remarkable result derived in [4] is that for the quasistatic, flat Rayleigh fading channel the best space-time codes (STC) may be obtained by optimizing two design criteria, namely, the rank criterion and the determinant criterion. Some STC for two transmit antennas for the 4-PSK and 8PSK modulations were provided in [4]. The STC presented in [4], and those presented subsequently in $[5,6]$, were either hand designed or found by exhaustive computer search. The main difficulty for designing STC is that the two aforementioned criteria apply to the complex field of baseband modulated signals rather than a discrete domain (e.g., the binary Galois field) in which the underlying codes could be designed. Recently, important papers providing general design procedures of STC were written by Hammons and El Gamal $[7,8]$, by Blum $[9,10]$, and by Grimm et. al. [6]. In [7, 8], general binary rank criteria (in substitution to the complexbased rank criteria) were developed that are simpler and yet sufficient to guarantee that the associated STC achieve maximum diversity for any number of transmit antennas. Results are valid for the BPSK and QPSK modulations. Blum's papers deal with binary convolutional codes that serve as STC. Necessary and sufficient conditions are given in $[9,10]$ for the 


\section{Mário de Noronha-Neto, Richard Demo Souza, and Bartolomeu F. Uchôa-Filho}

Space-Time Convolutional Codes Over GF $(p)$ for the Quasi-Static, Flat Rayleigh Fading Channel

STC to achieve maximum diversity gain. Also, Blum developed methods for calculating and bounding the coding gain (related to the determinant criterion). In [6] the design rules proposed in [4] for the case of two transmit antennas are generalized to the multiple antennas case through the concept of "zeros symmetry". In all the previous papers, the underlying codes were either binary or over an extended binary field.

In this paper, an alternative design procedure for achieving diversity and coding advantages is presented. We consider a space-time coded system consisting of a rate $R=1 / n$ linear convolutional encoder over $\mathrm{GF}(p)$, where $p$ can be any prime number, followed by a serial-to-parallel converter, $n$ mappers from $\mathrm{GF}(p)$ into a $p$-ary signal constellation (not necessarily PSK), and a transmitter with $n$ transmit antennas. This approach differs from those in the papers referenced above in that here the coded symbols are in one-to-one correspondence to the signals from the constellations. This makes the code design and the code search much easier to cope with. A spectral efficiency of $\log _{2}(p) \mathrm{b} / \mathrm{s} / \mathrm{Hz}$ is achieved. Such nonintegral rates may be useful to serve a wider range of multimedia applications. Although the case of multiple transmit antennas can be considered (by means of Grimm's "zeros symmetry"), we specialize in this paper to the case of two transmit antennas. Simple conditions on the generator matrices of a rate $R=1 / 2$ convolutional code are given so that maximum diversity advantage is guaranteed. Under these conditions, and due to the linear structure of the convolutional codes, the computer search effort with respect to the determinant criterion is dramatically reduced. In this regard, some properties of reciprocal STC's are derived. New space-time codes with two transmit antennas are presented for the 5-PSK and 7-PSK modulations, with spectral efficiencies 2.32 and $2.81 \mathrm{~b} / \mathrm{s} / \mathrm{Hz}$, respectively.

This paper is organized as follows. The space-time system model and the two design criteria for the quasi-static, flat Rayleigh fading channel as presented in [4] are summarized in Section 2. In Section 3, space-time convolutional codes (STCC) over $\mathrm{GF}(p)$ are introduced, and sufficient conditions on the generator matrices of a rate $R=1 / 2$ convolutional code are given so that maximum diversity advantage is guaranteed. Some examples of new STCC over GF(5) and GF(7) with encoder memories 1 and 2, and some properties of reciprocal STC's are presented in Section 4. Simulation results in the form of frame error rate versus signal-to-noise ratio are reported, in Section 5, for these codes for $m=1,2,3$ receive antennas. The corresponding coding and diversity advantages are verified, in agreement with the theoretical results presented in Section 3. Finally, in Section 6 we draw some final comments.

\section{SYSTEM MODEL}

We consider a communication system employing $n$ transmit antennas and $m$ receive antennas, as shown in Fig. 1. At the transmitter, data is first encoded by a rate $R=1 / n$ convolutional encoder over $\mathrm{GF}(p), p$ a prime, whose output is divided into $n$ parallel streams. These streams are mapped into a $p$-ary signal constellation and transmitted simultaneously from the $n$ antennas. A space-time system has been

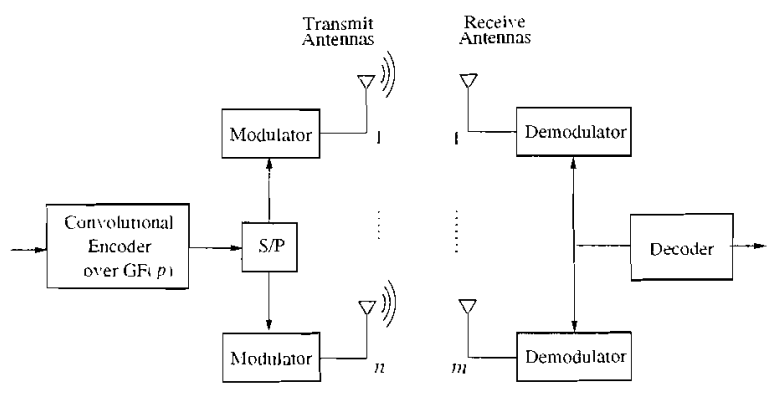

Figure 1. Space-time system model.

modeled by Tarokh et. al. [4] in such a way that the signal received by the $j$ th antenna at time $t, d_{t}^{j}$, is given by

$$
d_{t}^{j}=\sum_{i=1}^{n} a_{i, j} c_{t}^{i} \sqrt{E_{s}}+\eta_{t}^{j}
$$

where $c_{t}^{i}$ is the signal transmitted from the $i$ th antenna at time $t, E_{S}$ is the average energy of the transmitted signal, $\eta_{t}^{j}$ is a zero-mean complex white Gaussian noise with variance $N_{0} / 2$ per dimension, and $\alpha_{i, j}$ denotes the fade present in the path from the $i$ th transmit antenna to the $j$ th receive antenna. Under the Rayleigh fading assumption, the fades $\alpha_{i, j}$ for $1 \leq i \leq n$ and $1 \leq j \leq m$, are modeled as independent samples of a zero-mean complex Gaussian random process with variance 0.5 per dimension. In practice, to achieve independent fades the antennas must be separated by a few wavelengths. For the quasi-static, flat-fading channel, it is assumed that the path fades remain constant during a frame and change independently from one frame to another. Also, assume the receiver perfectly knows the channel state information (i.e., the $\alpha$ 's) and that the Viterbi algorithm with the Euclidean metric is used in the decoder.

\subsection{DESIGN CRITERIA}

In this section, we briefly review the derivation of the design criteria for slow Rayleigh fading channels [4]. For the system in Fig. 1, and described by (1), the conditional pairwise error probability, defined as the probability that a maximum-likelihood decoder decides erroneously in favor of codeword

$$
\mathbf{e}=e_{1}^{1} e_{1}^{2} \cdots e_{1}^{n} e_{2}^{1} \epsilon_{2}^{2} \cdots e_{2}^{n} \cdots e_{l}^{1} e_{l}^{2} \cdots e_{l}^{n}
$$

when the correct codeword is:

$$
\mathbf{c}=c_{1}^{1} c_{1}^{2} \cdots c_{1}^{n} c_{2}^{1} c_{2}^{2} \cdots c_{2}^{n} \cdots c_{l}^{1} c_{l}^{2} \cdots c_{l}^{n},
$$

given a fixed set of fades, can be approximated by:

$$
\begin{array}{r}
P\left(\mathbf{c} \rightarrow \mathbf{e} \mid \alpha_{i, j}, i=1,2, \ldots, n, j=1,2, \ldots, n\right) \\
\leq \exp \left(-d^{2}(\mathbf{c}, \mathbf{e}) E_{s} / 4 N_{0}\right)
\end{array}
$$

where $d^{2}(\mathbf{c}, \mathbf{e})$ is the square Euclidean distance between the sequences $\mathbf{c}$ and $\mathbf{e}$, and is given by:

$$
d^{2}(\mathbf{c}, \mathbf{e})=\sum_{j=1}^{m} \sum_{t=1}^{l}\left|\sum_{i=1}^{n} a_{i, j}\left(c_{t}^{i}-e_{t}^{i}\right)\right|^{2} .
$$


After some algebraic manipulations, (3) can be written as:

$$
d^{2}(\mathbf{c}, \mathbf{e})=\sum_{j=1}^{m} \Omega_{j} A(\mathbf{c}, \mathbf{e}) \Omega_{j}^{H}
$$

where $\Omega_{j}=\left(\alpha_{1, j}, \alpha_{2, j}, \ldots, \alpha_{n, j}\right)$ and $\{A(\mathbf{c}, \mathbf{e})\}_{p q}=$ $\sum_{t=1}^{l}\left(c_{t}^{p}-e_{t}^{p}\right)\left(c_{t}^{q}-\epsilon_{t}^{q}\right)^{*}$, for $1 \leq p, q \leq n$. Here the Hermitian transposition is denoted by $H$ and the complex conjugate by ${ }^{*}$. Note that the matrix $A(\mathbf{c}, \mathbf{e})$ is Hermitian and can be factored as $B(\mathbf{c}, \mathbf{e}) B(\mathbf{c}, \mathbf{e})^{H}$, where

$$
B(\mathbf{c}, \mathbf{e}) \triangleq\left(\begin{array}{cccc}
\epsilon_{1}^{1}-c_{1}^{1} & e_{2}^{1}-c_{2}^{1} & \cdots & e_{l}^{1}-c_{l}^{1} \\
e_{1}^{2}-c_{1}^{2} & e_{2}^{2}-c_{2}^{2} & \cdots & e_{l}^{2}-c_{l}^{2} \\
\vdots & \vdots & \ddots & \vdots \\
e_{1}^{n}-c_{1}^{n} & e_{2}^{n}-c_{2}^{n} & \cdots & e_{l}^{n}-c_{l}^{n}
\end{array}\right)
$$

is a square root of matrix $A(\mathbf{c}, \mathbf{e})$. According to [11], if a matrix $A$ with a square root $B$ is Hermitian then the eigenvalues of $A(\mathbf{c}, \mathbf{e})$ are non-negative real numbers.

Now, we write $d^{2}(\mathbf{c}, \mathbf{e})$ in terms of the eigenvalues of the matrix $A(\mathbf{c}, \mathbf{e})$. From linear algebra [1 1], for each Hermitian matrix $A$ there exists a unity matrix $V$ and a real diagonal matrix $D=\operatorname{diag}\left(\lambda_{1}, \lambda_{2}, \ldots, \lambda_{n}\right)$ such that $V A V^{H}=D$, where $\left(\lambda_{1}, \lambda_{2}, \ldots, \lambda_{n}\right)$ are the eigenvalues of $A(\mathrm{c}, \mathrm{e})$. Defining $\left(\beta_{1, j}, \beta_{2, j}, \ldots, \beta_{n, j}\right)=\Omega_{j} V^{*}$, we arrive after some algebraic manipulation at the following expression for the upper bound in (2):

$$
\prod_{j=1}^{m} \exp \left(-\left(E_{s} / 4 N_{0}\right) \sum_{i=1}^{n} \lambda_{i}\left|\beta_{i, j}\right|^{2}\right) .
$$

Since the $\alpha_{i, j}$ 's are modeled as independent samples of a zero-mean complex Gaussian random process with variance 0.5 per dimension, and $V$ is unitary, then the $\beta_{i, j}$ 's are also independent zero-mean complex Gaussian random variables with variance 0.5 per dimension. Therefore, we can calculate the average value of (5) and obtain, after some simplifications based on the large signal-to-noise ratios assumption, an upper bound on the average pairwise error probability, given by:

$$
P(\mathbf{c} \rightarrow \mathbf{e}) \leq\left(\prod_{i=1}^{r} \lambda_{i}\right)^{-m}\left(\frac{E_{s}}{4 N_{0}}\right)^{-r m}
$$

where $r$ is the rank of the matrix $B(\mathbf{c}, \mathbf{e})$ and $\lambda_{i}, i=1, \ldots, r$, are the nonzero eigenvalues of $A(\mathbf{c}, \mathbf{e})$.

Based on (6), Tarokh et. al. [4] arrived at two design criteria for the quasi-static, flat Rayleigh fading channel, namely,

- The Rank Criterion: In this criterion the parameter to be maximized is the minimum rank $r$ of matrix $B(\mathbf{c}, \mathbf{e})$, over all distinct pairs of codewords $\mathbf{c}$ and $\mathbf{e}$. It is said that this is an $r$-space-time code. The diversity advantage is $r m \leq n m$, with equality at full rank condition, that is, $r=n$.

- The Determinant Criterion: For a given diversity advantage $r m$, the goal of this criterion is to maximize the minimum geometric mean of the nonzero eigenvalues of the matrix $A(\mathbf{c}, \mathrm{e}),\left(\lambda_{1} \lambda_{2} \ldots \lambda_{r}\right)^{\frac{1}{r}}$, over all distinct pairs of codewords $c$ and $e$. This represents the coding gain.

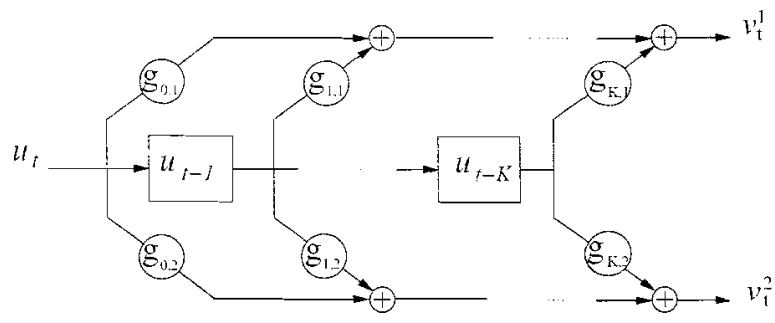

Figure 2. Rate 1/2 convolutional encoder over $\mathrm{GF}(p)$.

In the next section we derive conditions on the generator matrix of a rate $R=1 / 2$ convolutional code so that maximum diversity advantage, as presented in Section 2, is guaranteed for the corresponding 2-space-time codes.

\section{SPACE-TIME CONVOLUTIONAL CODES OVER GF(p)}

Let $U(D)=u_{0}+u_{1} D+u_{2} D^{2}+\cdots$ be the polynomial over $\mathrm{GF}(p), p$ a prime, representing a data sequence. This sequence is encoded by a rate $R=1 / n$ convolutional encoder over $\operatorname{GF}(p)$, which is the direct realization of the polynomial generator vector:

$$
\mathbf{G}(D)=\left[G_{1}(D), G_{2}(D), \ldots, G_{n}(D)\right]
$$

producing the encoded vector

$$
\mathbf{V}(D)=U(D) \mathbf{G}(D)=\left[V^{1}(D), V^{2}(D), \ldots, V^{n}(D)\right]
$$

where $V^{i}(D)=v_{0}^{i}+v_{1}^{i} D+v_{2}^{i} D^{2}+\cdots+v_{t}^{i} D^{t}+\cdots$, for $i=1,2 \ldots, n$, are the $n$ encoded sequences. The code generators are $G_{i}(D)=g_{0, i}+g_{1, i} D+g_{2, i} D^{2} \cdots+g_{K, i} D^{K}$, for $i=1,2 \ldots, n$, where $K$ is the memory of the encoder. An example of a rate $1 / 2$ convolutional encoder over $G F(p)$ is shown in Fig. 2.

At each time $t$, a data symbol from $\mathrm{GF}(p)$ produces a block of $n$ symbols from $\mathrm{GF}(p)$ denoted by $\left(v_{t}^{1}, v_{t}^{2}, \ldots, v_{t}^{n}\right)$. Regarding the space-time system model in Fig. 1, these symbols are mapped into a $p$-ary signal constellation, as indicated in Fig. 3 for the 5-PSK and 7-PSK constellations. The $n$ complex symbols, $\left(c_{t}^{1}, c_{t}^{2}, \ldots, c_{t}^{n}\right)$, are then transmitted by the $n$ antennas. A sequence of blocks $\left(c_{t}^{1}, c_{t}^{2}, \ldots, c_{t}^{n}\right)$, for $t=1,2, \ldots, l$ forms a codeword $\mathbf{c}$ of the space-time code.

From now on, we shall focus our attention on the case of $n=2$ transmit antennas. Thus, according to Tarokh's design criteria, the code search should aim at finding the "full rank" code $\mathcal{C}$ that maximizes the minimum determinant

$$
\operatorname{det}\left(\sum_{i=1}^{l}\left(e_{i}^{1}-c_{i}^{1}, e_{i}^{2}-c_{i}^{2}\right)^{*}\left(e_{i}^{1}-c_{i}^{1}, e_{i}^{2}-c_{i}^{2}\right)\right),
$$

over all pairs of codewords $\mathbf{c}$ and $\mathbf{e}$, where ${ }^{*}$ denotes complex conjugate. In the following, we explore the algebraic structure of the convolutional codes and present some guidelines to guarantee the full rank condition, yielding a reduced complexity code search. 
Lemma 1 Consider a rate $R=1 / 2$ convolutional spacetime code over $G F(p)$, p a prime, with a generator matrix

$$
\mathrm{G}(D)=\left[\begin{array}{c}
g_{1.1} D+\ldots+g_{K^{i} 1} D^{K^{-}} \\
g_{0.2}+\ldots+g_{K-1.2} D^{K^{-}-1}
\end{array}\right]^{T},
$$

where $g_{h .1} \neq 0$ and $g_{0.2} \neq 0$. Then, 1 ) all branches in the code trellis departing from the same state coincide in the first symbol and differ in the second symbol, and 2) all transitions arriving at the same state coincide in the second symbol and differ in the first symbol.

Proof: We shall use the fact that, for field elements $u$, $u^{\prime}$, and $g$ :

$$
u \cdot g=u^{\prime} \cdot g \quad \Rightarrow \quad u=u^{\prime},
$$

and

$$
u \cdot g \neq u^{\prime} \cdot g \Rightarrow u \neq u^{\prime} .
$$

To prove 1 ), let us consider the current state as being $\sigma_{s}=$ $\left[u_{1}, \ldots, u_{K^{-}}\right]^{1}$. When $u_{0}(f)$ is the input symbol, the state transition is $\sigma_{s} \rightarrow \sigma_{f}=\left[u_{o}(f), u_{1}, \ldots, u_{K^{*}-1}\right]$. Since $g_{0.1}=0$, the coded symbol corresponding to the first antenna will be given by:

$$
v^{1}=\sum_{\mu=1}^{K-1} u_{\mu} \cdot g_{\mu, 1}+u_{K} \cdot g_{K, 1},
$$

which is a constant in GF( $(p)$ that does not depend on $u_{0}(f)$, but on $\sigma_{s}$. This shows that the first symbol of all branches departing from the same state are equal. Now for the same state transition $\sigma_{s} \rightarrow \sigma_{f}$, since $g_{K^{2}, 2}=0$, the symbol corresponding to the second antenna will be given by:

$$
v^{2}=u_{0}(f) \cdot g_{0,2}+\sum_{\mu=1}^{K-1} u_{\mu} \cdot g_{\mu, 2}=u_{0}(f) \cdot g_{0.2}+V_{2}
$$

where $V_{2}$ is a constant in $\mathrm{GF}(p)$. Thus, in $\mathrm{GF}(p), v^{2}$ will be different for different values of $u_{0}(f)$.

To prove 2), let us consider the final state as being $\sigma_{f}=$ $\left[u_{0}, u_{1}, \ldots, u_{K^{\prime}-1}\right]$. Then, for the state transition $\sigma_{s}=$ $\left[u_{1}, \ldots, u_{K^{-1}}, u_{K^{\prime}}(s)\right] \rightarrow \sigma_{f}$, the symbol corresponding to the first antenna is given by:

$$
v^{1}=\sum_{\mu=1}^{K-1} u_{\mu} \cdot g_{\mu, 1}+u_{K^{*}}(s) \cdot g_{K^{*}, 1}=V_{1}+u_{K^{K}}(s) \cdot g_{K, 1},
$$

where $V_{1}$ is a constant in $\mathrm{GF}(p)$. So, in $\mathrm{GF}(p), v^{1}$ will be different for different values of $u_{K}(s)$. For the same transition $\sigma_{s} \rightarrow \sigma_{f}$, the symbol corresponding to the second antenna will be given by:

$$
v^{2}=u_{0} \cdot g_{0,2}+\sum_{\mu=1}^{K-1} u_{\mu} \cdot g_{\mu, 2},
$$

which is a constant in $\operatorname{GF}(p)$ that does not depend on $u_{K}(s)$, only on $\sigma_{f}$. This completes the proof. ity.

${ }^{l}$ Note that here we drop the index $t$ used in Fig. 2 for the sake of simplic-

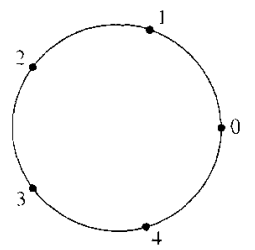

(a)

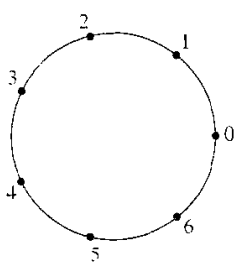

(b)

Figure 3. Constellations 5-PSK (a) and 7-PSK (b).

Theorem 1 Consider a rate $R=1 / 2$ convolutional spacetime code over $G F(p)$, $p$ a prime. If the generator matrix $\mathbf{G}$ is in accordance with Lemma I, then the full rank condition is guaranteed.

Proof: Assume the generator matrix G is in accordance with Lemma 1 , and consider an arbitrary pair of codewords $\mathbf{c}$ and $\mathbf{e}$ of length $2 l$ symbols. Then, equation (7) results in:

$$
\operatorname{det}_{l} \triangleq \operatorname{det}\left(\left[\begin{array}{cc}
0 & 0 \\
0 & \left|a_{1}\right|^{2}
\end{array}\right]+\left[\begin{array}{cc}
f & d \\
d^{*} & h
\end{array}\right]+\left[\begin{array}{cc}
\left|b_{l}\right|^{2} & 0 \\
0 & 0
\end{array}\right]\right)
$$

where

$$
\begin{gathered}
a_{i}=\left(e_{i}^{2}-c_{i}^{2}\right), \quad b_{i}=\left(e_{i}^{1}-c_{i}^{1}\right), \quad f=\sum_{i=2}^{l-1}\left|b_{i}\right|^{2}, \\
h=\sum_{i=2}^{l-1}\left|a_{i}\right|^{2}, \quad \text { and } \quad d=\sum_{i=2}^{l-1} a_{i} b_{i}^{*} .
\end{gathered}
$$

From Lemma 1, we have that $\left|a_{1}\right|^{2}>0$ and $\left|b_{l}\right|^{2}>0$. By Schwarz inequality,

$$
\sum_{i}\left|a_{i}\right|^{2} \times \sum_{i}\left|b_{i}\right|^{2} \geq\left|\sum_{i} a_{i} b_{i}^{*}\right|^{2}
$$

and thus $f h \geq|d|^{2}$. Since $f \geq 0$ and $h \geq 0$, we must have that:

$$
\operatorname{det}_{l}=\underbrace{\left|a_{1}\right|^{2}\left|b_{l}\right|^{2}}_{>0}+\underbrace{\left|a_{1}\right|^{2} f}_{\geq 0}+\underbrace{\left|b_{l}\right|^{2} h}_{\geq 0}+\underbrace{f h-|d|^{2}}_{\geq 0}>0,
$$

which guarantees the full rank condition.

\section{CODE SEARCH RESULTS}

In this section, we present some examples of 2-space-time codes generated by a rate $R=1 / 2$ linear convolutional encoder over $\mathrm{GF}(5)$ and $\mathrm{GF}(7)$. The restrictions derived in the previous section are applied to the convolutional encoder (i.e., $g_{K^{i}, 1} \neq 0, g_{0,2} \neq 0$ and $g_{0,1}=g_{K .2}=0$ ) to guarantee the full rank condition. The minimum determinant is then maximized. We begin with examples of unit-memory encoders.

Example 1 Consider the 5-PSK constellation in Fig. 3(a). Following the restrictions of the previous section, the unitmemory convolutional encoder over $G F(5)$ has generator matrix $\mathbf{G}(D)=\left[g_{1.1} D, g_{0,2}\right]$. These codes have diversity gain 2 , with spectral efficiency of $2.32 \mathrm{~b} / \mathrm{s} / \mathrm{H}_{\xi}$. In performing 


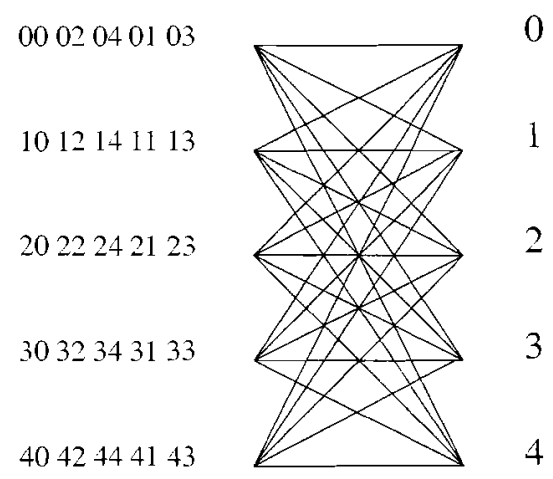

Figure 4. 2-space-time code for 5 -PSK, $2.32 \mathrm{~b} / \mathrm{s} / \mathrm{Hz}$, (convolutional encoder: $\mathrm{GF}(5), R=1 / 2, K=1$ ).

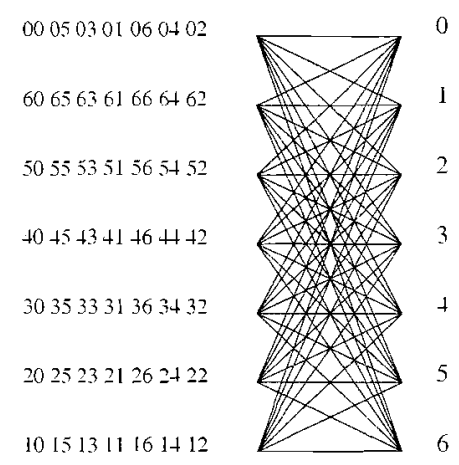

Figure 5. 2-space-time code for 7-PSK, $2.81 \mathrm{~b} / \mathrm{s} / \mathrm{Hz}$, (convolutional encoder: $\mathrm{GF}(7), R=1 / 2, K=1$ ).

a code search, we made $g_{1.1}$ and $g_{0.2}$ to vary over all nonsero elements in $G F(5)$, resulting in 16 different codes. The minimum determinant of $A(\mathbf{c}, \mathbf{e})$ for each code is listed in the matrix $D\left(g_{1.1}, g_{0,2}\right)$ below:

$$
D\left(g_{1,1}, g_{0,2}\right)=\left(\begin{array}{llll}
1,38 & 2,23 & 2,23 & 1,38 \\
2,23 & 1,38 & 1,38 & 2,23 \\
2,23 & 1,38 & 1,38 & 2,23 \\
1,38 & 2,23 & 2,23 & 1,38
\end{array}\right),
$$

where $g_{1,1}$ is the row index and $g_{0,2}$ is the column index.

Clearly, the best code is obtained with, for instance, $g_{1.1}=$ 1 and $g_{0.2}=2$. This code has coding gain 2.23. The trellis of this code is shown in Fig.4.

In this and in the following trellises, each pair of symbols to the left of the trellis indicates the signal transmitted over the first and the second antennas, respectively, and the label to the right of the trellis indicates the state of the encoder.

Example 2 Now consider the signal constellation 7-PSK in Fig. 3(b). Again, we consider a unit-memory encoder satisfying the conditions of Section 3. We thus have diversity gain 2, with spectral efficiency of $2.81 \mathrm{~b} / \mathrm{s} / \mathrm{H}$ - Similarly to Example 1 , we obtained the matrix $D\left(g_{1,1}, g_{0,2}\right)$ as

$$
D\left(g_{1.1, g_{0,2}}\right)=\left(\begin{array}{llllll}
0,75 & 1,35 & 1,35 & 1,35 & 1.35 & 0.75 \\
1.35 & 0.75 & 1.35 & 1.35 & 0.75 & 1,35 \\
1.35 & 1.35 & 0.75 & 0.75 & 1.35 & 1,35 \\
1.35 & 1.35 & 0.75 & 0.75 & 1.35 & 1,35 \\
1,35 & 0.75 & 1.35 & 1.35 & 0.75 & 1,35 \\
0.75 & 1,35 & 1,35 & 1.35 & 1.35 & 0,75
\end{array}\right)
$$

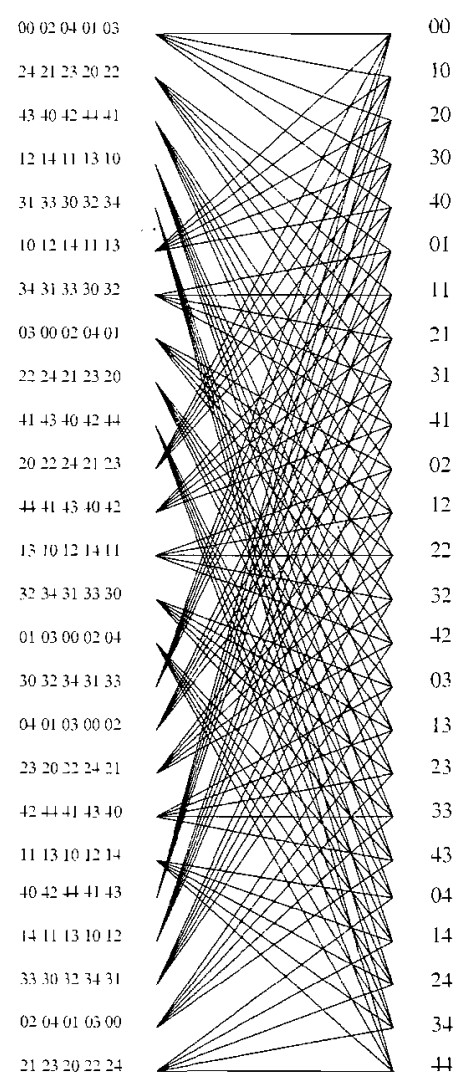

Figure 6. 2-space-time code for 5 -PSK, $2.32 \mathrm{~b} / \mathrm{s} / \mathrm{Hz}$, (convolutional encoder: $\mathrm{GF}(5), R=1 / 2, K^{\prime}=2$ ).

The best code is obtained with, for instance, $g_{1.1}=6$ and $g_{0,2}=5$. This code has coding gain 1.35. The trellis of this code is shown in Fig.5.

Example 3 Here we extend Example 1 to the case of volutional encoder of memory order $K=2$ over $G F(5)$. L nut is, the convolutional encoder has generator matrix $\mathbf{G}(D)=$ $\left[g_{1,1} D+g_{2.1} D^{2}, g_{0.2}+g_{1,2} D\right]$. For this case, one best code is generated by $g_{1.1}=2, g_{2,1}=1, g_{0.2}=2$, and $g_{1,2}=4$ (see trellis in Fig. 6). The coding gain of this code is 4.47 . The diversity gain and the spectral efficiency are the same as those in Example 1.

Example 4 In this example, we consider a convolutional encoder of memory order $K=2$ over $G F(7)$. Similarly to Example 2, the generator matrix is $\mathbf{G}(D)=\left[g_{1.1} D+\right.$ $\left.g_{2.1} D^{2}, g_{0.2}+g_{1.2} D\right]$. The multipliers set as $g_{1.1}=3$, $g_{2,1}=2, g_{0,2}=1$, and $g_{1.2}=3$ produce one best code. The trellis description for this code is shown in Fig. 7. The coding gain is 3.74, while the diversity gain and the spectral efficiency are the same as those in Example 2.

It should be noticed that the two matrices $D\left(g_{1,1}, g_{0.2}\right)$ above, for the cases of unit-memory convolutional codes over $\mathrm{GF}(5)$ and $\mathrm{GF}(7)$, are symmetric. We prove next that such a symmetry holds true for any field $\mathrm{GF}(p), p$ a prime, and any encoder memory $K$. This fact can be used to limit the code 


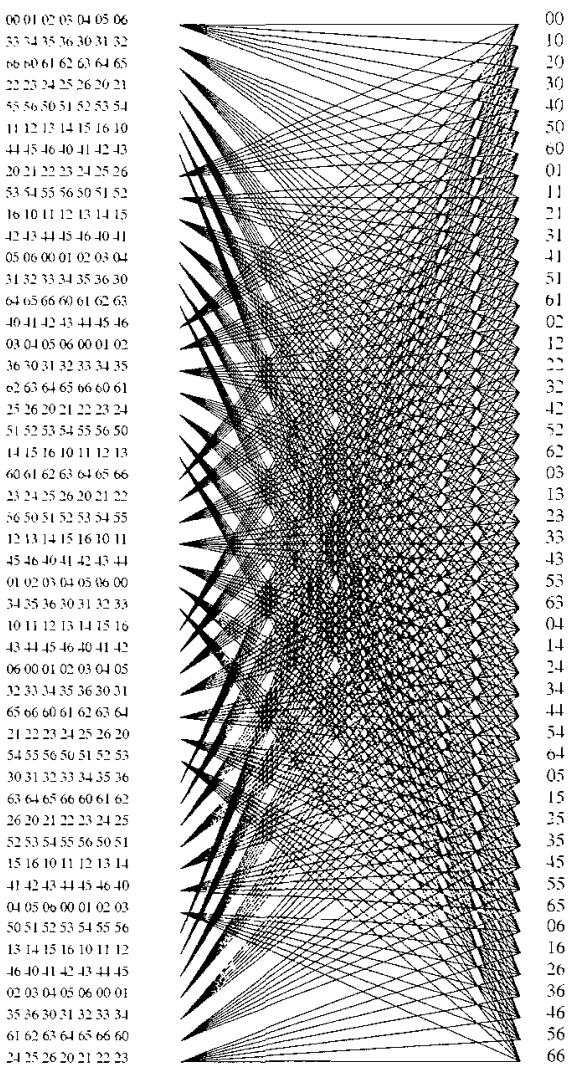

Figure 7. 2-space-time code for 7-PSK, $2.81 \mathrm{~b} / \mathrm{s} / \mathrm{Hz}$, (convolutional encoder: $\mathrm{GF}(7), R=1 / 2, K^{\circ}=2$ ).

search even further, for the cases of larger values of $p$ and $K$.

Theorem 2 Consider the space-time system model in Fig. 1. Let $\mathbf{G}(D)=\left[G_{1}(D), G_{2}(D)\right]$ be the generator matrix of a rate $R=1 / 2$ convolutional code over $G F(p), p$ a prime, with code generators $G_{1}(D)=g_{0.1}+g_{1.1} D+$ $g_{2.1} D^{2} \cdots+g_{K, 1} D^{K}$ and $G_{2}(D)=g_{0.2}+g_{1.2} D+$ $g_{2.2} D^{2} \cdots+g_{K, 2} D^{K}$. The space-time convolutional code generated by $\mathrm{G}(D)$ has the same diversity gain and coding gain as the space-time convolutional code generated by the generator matrix $\hat{\mathbf{G}}(D)=\left[\hat{G}_{1}(D), \hat{G}_{2}(D)\right]$, with code generators $\hat{G}_{1}(D) \triangleq D^{K} G_{1}\left(D^{-1}\right)=g_{K^{-} .2}+g_{K-1,2} D+$ $g_{K-2,2} D^{2} \cdots+g_{0,2} D^{K}$ and $\hat{G}_{2}(D) \triangleq D^{K} G_{2}\left(D^{-1}\right)=$ $g_{K, 1}+g_{K-1,1} D+g_{K-2.1} D^{2} \cdots+g_{0,1} D^{K^{\prime}}$

Proof: Consider the convolutional encoder of Fig. 2, for the code generators $G_{1}(D)$ and $G_{2}(D)$. Let the current state be $\sigma_{s}=\left[u_{1}, u_{2}, \ldots, u_{K}\right]$, where $u_{i} \in \mathrm{GF}(p)$, $i=1,2, \cdots, K$. The input symbol $u_{o}$ causes the state transition $\sigma_{s} \rightarrow \sigma_{f}=\left[u_{o}, u_{1}, \ldots, u_{K-1}\right]$ and the coded outputs, $v^{1}$ and $v^{2}$, are:

$$
v^{1}=\sum_{\mu=0}^{K} u_{\mu} \cdot g_{\mu, 1}
$$

and

$$
v^{2}=\sum_{\mu=0}^{K} u_{\mu} \cdot g_{\mu, 2} .
$$

For the code generators $\hat{G}_{1}(D)$ and $\hat{G}_{2}(D)$, suppose that the current state is $\hat{\sigma}_{s}=\left[\hat{u}_{1}, \hat{u}_{2}, \ldots, \hat{u}_{F^{-}}\right]$. In this case. the input symbol $\hat{u}_{o}$ causes the state transition $\hat{\sigma}_{s} \rightarrow \hat{\sigma}_{f}=$ $\left[\hat{u}_{0}, \hat{u}_{1}, \ldots, \hat{u}_{K-1}\right]$ and the coded outputs, $\hat{v}^{1}$ and $\hat{v}^{2}$, are:

$$
\hat{v}^{1}=\sum_{\mu=0}^{\hbar^{-}} \hat{u}_{\mu} \cdot g_{K-\mu .2}
$$

and

$$
\hat{v}^{2}=\sum_{\mu=0}^{K} \hat{u}_{\mu} \cdot g_{K-\mu .1} .
$$

When $u_{2}=\hat{u}_{K^{r}-i}$, for $i=0,1,2, \ldots, K$, the coded outputs $\hat{v}^{1}$ and $\hat{v}^{2}$ are, respectively, $r^{2}$ and $r^{1}$, and the corresponding state transition is $\hat{\sigma}_{s}=\left[u_{I_{s}-1}, \ldots, u_{1}, u_{0}\right] \rightarrow \hat{\sigma}_{f}=$ $\left[u_{K}, \ldots, u_{2}, u_{1}\right]$. The code generated by $\hat{\mathbf{G}}(D)$ is called the reciprocal code of the code generated by $\mathbf{G}(D)$. It follows that for every pair of space-time codewords

$$
\mathbf{c}=c_{1}^{1} c_{1}^{2} \cdots c_{1}^{n} c_{2}^{1} c_{2}^{2} \cdots c_{2}^{n} \cdots c_{l}^{1} c_{l}^{2} \cdots c_{l}^{n},
$$

and

$$
\mathrm{e}=e_{1}^{1} e_{1}^{2} \cdots e_{1}^{n} e_{2}^{1} e_{2}^{2} \cdots e_{2}^{n} \cdots e_{l}^{1} e_{l}^{2} \cdots e_{l}^{n}
$$

in the original code, there is a pair of space-time codewords

$$
\hat{\mathbf{c}}=c_{l}^{n} \cdots c_{l}^{2} c_{l}^{1} \cdots c_{2}^{n} \cdots c_{2}^{2} c_{2}^{1} c_{1}^{n} \cdots c_{1}^{2} c_{1}^{1},
$$

and

$$
\hat{\mathbf{e}}=e_{l}^{n} \cdots e_{l}^{2} e_{l}^{1} \cdots e_{2}^{n n} \cdots e_{2}^{2} e_{2}^{1} e_{1}^{n} \cdots e_{1}^{2} e_{1}^{1},
$$

in the reciprocal code. Since both pairs of codewords yield matrices $B(\mathbf{c}, \mathrm{e})$ with the same rank and the same determinant, the two space-time codes must have the same diveristy gain and coding gain.

\section{SIMULATION RESULTS}

In this section, we present simulation results for the four 2 -space-time codes designed in the last section. We consider the cases of $m=1,2,3$ receive antennas and frame length $l=130$ symbols.

Figure 8 shows the frame error rate (FER) versus the signal-to-noise ratio (SNR), in decibels, for the codes constructed over $G F(5)$, with $K=1,2$. As we can predict from (6), for the same number of receive antennas, the code with memory order $K=2$, and coding gain 4.47 , has a better performance than the code with unitary memory, and coding gain 2.23. With the increase in the number of receiving antennas, the performance is ameliorated for both cases, $K=1,2$, while the relative advantage of the code with memory order $K=2$ is magnified. Again, this behavior can be induced from (6).

In Figure 9 we have the same comparisons as in Figure 8 , but this case for the codes constructed over $G F(7)$. As in Figure 8 , the results are in accordance with (6), what validates our code construction method and search results.

\section{FINAL COMIMENTS}

In this paper, we considered space-time convolutional codes over $\mathrm{GF}(p)$ for the quasi-static, flat Rayleigh channel. 


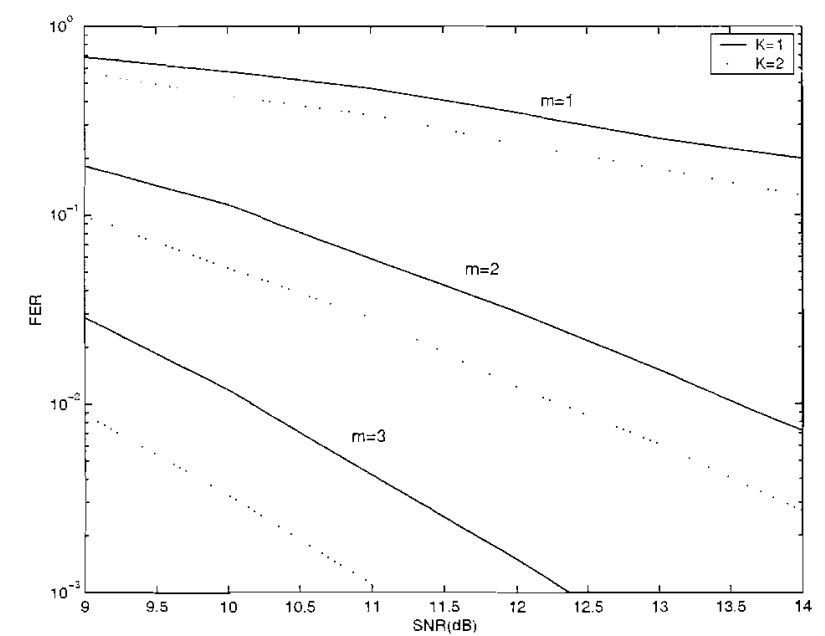

Figure 8. Frame error rate (FER) versus signal-to-noise ratio (SNR) for the 2-space-time codes for 5-PSK with $K=1,2$ and $m=1,2,3$ receive antennas.

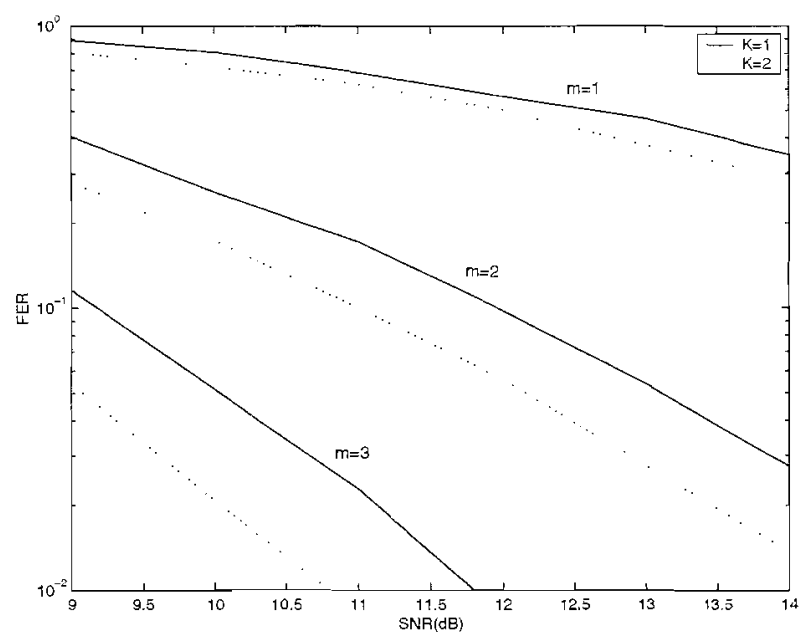

Figure 9. Frame error rate (FER) versus signal-to-noise ratio (SNR) for the 2-space-time codes for 7-PSK with $K=1,2$ and $m=1,2,3$ receive antennas.

The codes were designed to provide the best performance according to the rank and the determinant criteria derived by Tarokh et. al.. Simple conditions on the generator matrices of a rate $1 / 2$ convolutional code were given that guarantee maximum diversity advantage for 2 transmit antennas.

New space-time convolutional codes for 2 transmit antennas for the 5-PSK and 7-PSK constellations were presented. Spectral efficiencies of 2.32 and $2.81 \mathrm{~b} / \mathrm{s} / \mathrm{Hz}$, respectively, were achieved, providing more options than the usual 2 and $3 \mathrm{~b} / \mathrm{s} / \mathrm{Hz}$

Simulation results in the form of frame error rate versus signal-to-noise ratio were reported for these codes for $m=1,2,3$ receive antennas. The corresponding coding and diversity advantages were verified, in agreement with the theoretical results presented in Section 3.

The space-time codes presented in this paper could be found with a reduced computer search, due to the linearity of the convolutional codes, the restrictions derived in Section 3, and the symmetry of the matrix $D$ in Section 4 . Specifically, an exhaustive code search for the cases of the examples in Section 4 would need to check a huge number $\left(O\left(p^{\left(p^{K} 2 p\right)}\right)\right)$ of codes. By considering the convolutional codes which satisfy the conditions of Theorem 1 , one would need to check only $O\left(p^{2 K^{*}}\right)$ codes. The number of codes that have to be checked can be reduced further if one takes the symmetry of Theorem 2 into account. For instance, in Example 1 only 10 candidates were checked and, for the more complex case, in Example 4, there were 735 candidates. These advantages become more significant as the memory order increases, allowing good codes to be obtained with relatively low computer effort.

The fact that $p$ is not a power of 2 may pose as a problem if transmission of binary data is required. We tackle this problem by associating a different word of $n_{p} p$-ary symbols to each word of $n_{2}$ bits, where $p^{n_{p}}>2^{n_{2}}$. The spectral efficiency is $n_{2} / n_{p}<\log _{2}(p) \mathrm{b} / \mathrm{s} / \mathrm{Hz}$, that is, there is a percentual loss of

$$
\left[1-\left(\frac{n_{2}}{n_{p} \log _{2}(p)}\right)\right] \times 100 .
$$

Clearly this loss is minimized by maximizing the relation $\frac{n_{2}}{n_{p} \log _{2}(p)}$, where $p^{n_{p}}>2^{n_{2}}$. For instance, consider that, in the case of the 5-PSK constellation, each word of $n_{2}=9$ bits is associated to a different word of $n_{p}=4$ symbols. Then, the spectral efficiency will be $2.25 \mathrm{~b} / \mathrm{s} / \mathrm{Hz}$, producing a loss of only $3 \%$.

\section{REFERENCES}

[1] E. Telatar, "Capacity of multi-antenna Gaussian channels," AT\&T Bell Labs, Tech. Rep., June 1995.

[2] G. J. Foschini and M. Gans, "On the limits of wireless communication in a fading environment when using multiple antennas," Wireless Pers. Commun., vol. 6, pp. 311-335, Mar. 1998.

[3] T. Marzetta and B. Hochwald, "Capacity of a mobile multiple antenna communication link in Rayleigh flat fading," IEEE Trans. Inform. Theory, vol. 45, pp. 139-158, Jan. 1999.

[4] V. Tarokh, N. Seshadri, and A. R. Calderbank."Space-Time codes for high data rate wireless communication: performace criterion and code construction." IEEE Trans. on Inform. Theory, vol. 44, no. 2, pp. 744-765, Mar. 1998.

[5] S. Baro, G. Bauch, and A. Hansman, "Improved codes for space-time trellis coded modulation," IEEE Commun. Lett., pp. 20-22. Jan. 2000.

[6] J. Grimm, M. P. Fitz, and J. V. Krogmeier, "Further results in space-time coding for Rayleigh fading." in Proc. 36th Allerton Conf. on Communications, Control and Computing, Sept. 1998.

[7] A. R. Hammons, Jr. and H. El Gamal. "On the theory of spacetime codes for PSK modulation," IEEE Trans. on Inform. Theory, vol. 46, no. 2, pp. 524-542, Mar. 2000.

[8] H. El Gamal and A. R. Hammons, Jr., "On the design and performance of algebraic space-time codes for BPSK and QPSK modulation," IEEE Trans. on Commun., vol. 50, No. 6, pp. 907-913, June 2002.

[9] R. S. Blum, "New analytical tools for designing space-time convolutional codes," in Conf. Inform. Sci. Systm., Princeton, NJ, Mar. 2000. 
[10] R. S. Blum, "Some analytical tools for the design of spacetime convolutional codes," IEEE Trans. Conmunn., vol. 50. no. 10, pp. 1593-1599, Oct. 2002.

[11] R. A. Horn and C. R. Johnson, Matrix Analysis. New York: Cambridge Univ. Press, 1988.

Mário de Noronha-Neto was born in Jacarezinho - PR, Brazil, in 1977. He received the B.Sc. and the M.Sc. degrees in Electrical Engineering from the Federal University of Santa Catarina (UFSC), Florianópolis - SC, Brazil, in 2000 and 2002, respectively. He is currently working towards the D.Sc. degree in the same institution. His research interests are coding theory and wireless communications.

Richard Demo Souza was born in Florianópolis - SC. Brazil, in 1978. He received the B.Sc. degree in Electrical Engineering from the Federal University of Santa Catarina (UFSC), Florianópolis SC. Brazil, in 1999. He is currently working towards the D.Sc. degree in the same institution. His research interests are coding theory, wireless communications, and signal processing.
Bartolomeu F. Uchôa-Filho was born in Recife, Brazil, on October 29,1965 . He received the B.S.E.E. degree from the Federal University of Pernambuco (UFPE), Recife. Brazil, in 1989; the M.S.E.E. degree from the State University of Campinas (UNICAMP), Campinas, Brazil, in 1992; and the Ph.D. degree in electrical engineering from the University of Notre Dame, Notre Dame, Indiana. U.S.A., in 1996. During 1997-1999 he held a Post-Doctoral position at the State University of Campinas, where he taught graduate courses on information theory and digital communications. From August 1999 to January 2000 he was a Visiting Researcher in the Department of Electrical Engineering, Federal University of Santa Catarina, Florianópolis, Brazil, where he taught courses in mobile communications and telecommunications. Since February 2000 he has been an Associate Professor in the same Department. Dr. Uchôa has been serving as a technical reviewer for the IEEE Transactions on Information Theory, IEEE Transactions on Communications, and the Journal of the Brazilian Telecommunications Society. His research interests are in the area of coding and information theory, with applications to digital communications and information storage systems. Dr. Uchôa is a member of Eta Kappa Nu, IEEE Information Theory Society, and the Brazilian Telecommunications Society. 